\title{
AVANCES EN EL DISEÑO DE ESQUEMAS DE PAGOS POR SERVICIOS AMBIENTALES LOCALES EN LA AMAZONIA BAJA PERUANA: EL CASO DE LA CUENCA DEL NANAY
}

\author{
Javier Gustavo Montoya-Zumaeta'; José Luis Nolazco Cama²
}

Fecha de recepción: $\quad$ 04-03-15

\section{Resumen}

Se aplicó el Método de Valoración Contingente (MVC) para determinar la Disposición a Pagar (DAP) de los usuarios del servicio público de agua potable de lquitos para financiar un mecanismo tipo PSA para contribuir a la conservación de los ecosistemas que regulan la provisión del recurso hídrico. Los resultados muestran que la DAP mensual (promedio) por hogar asciende a $S / .1,77$ (US\$ 0.59) y $\mathrm{S} / .1,72$ (US\$ 0.57) utilizando especificaciones logit y probit respectivamente. Los valores en medianas, usando las técnicas no paramétricas de Kriström y Turnbull, se estimaron en $\mathrm{S} /$. 1,91 (US\$ 0.64) y S/ 1.69 (US\$ 0.56), respectivamente. Por lo tanto, se pudieron identificar los atributos de los potenciales contribuyentes al fondo de conservación, y generar evidencia empírica de la aplicación de los métodos de valoración económica en el contexto de la Amazonia baja peruana.

Palabras clave: Valoración Contingente, Iquitos, Disposición a Pagar, Nanay, Pagos por Servicios Ambientales.

\section{Clasificación JEL: Q52}

1 Magister en Socioeconomía Ambiental (Centro Agronómico Tropical de Investigación y Enseñanza, Costa Rica). Consultor World Agroforestry Centre. Dirección Postal: Carretera Federico Basadre Km. 4,2 (Ex-Cenfor) Pucallpa-Perú. Teléfono: +(51) 61579078. E-mail: jmontoya.iq@gmail.com.

Fecha de aceptación: 30-04-15

\section{Abstract}

It ${ }^{2}$ was applied the Contingent Valuation Method (CVM) to determine the willingness to pay (WTP) of public drinking water users in lquitos in order to fund a PES-like mechanism that contribute to conservation of ecosystems that regulate provision of the hydric resource. The results show monthly WTP (average) per household amounting to S/. 1,77 (US\$ 0.59) and S/. 1,72 (US\$ 0.57) using logit and probit specifications respectively. The values in medians using the nonparametric techniques of Kriström and Turnbull were estimated in S/.1,91 (US\$ 0.64) and S/. 1.69 (US\$ 0.56) respectively. Therefore it could be identified attributes of potential contributors to the conservation fund; and to generate empirical evidence of economic valuation methods' application in the Peruvian Amazon lowland's context.

Keywords: Contingent Valuation, Iquitos, WiIlingness to Pay, Nanay, Payment for Environmental Services.

JEL Calification: Q52

2 Economista (UNALM-Perú). Estudiante del Magister en Análisis Económico (Universidad de Chile, Chile). Dirección postal: Calle Helsinski 363, Ate-Vitarte, Lima-Perú. Teléfono: (511) 3511463. E-mail: jnolazco@fen.uchile.cl 


\section{INTRODUCCIÓN}

La cuenca amazónica continental brinda diversos servicios ecosistémicos que van desde el ámbito local como el abastecimiento de alimentos y medicinas, la regulación hidrológica y la polinización de cultivos; a otros servicios de escala global como la mitigación de emisiones de carbono y otros gases de efecto invernadero (Fearnside 1996). La Amazonía cubre más de las dos terceras partes del territorio peruano, y su importancia ecológica ha llevado a que aproximadamente la cuarta parte de su extensión total se encuentre establecida en Áreas Naturales Protegidas (ANP), históricamente la estrategia para su conservación más utilizada.

El principal desafío que amenaza la gestión de las ANP (e inherentemente los ecosistemas amazónicos dentro de estas áreas) es su sostenibilidad financiera (Hardner, 2008, Sernanp, 2009). En este sentido, los Pagos por Servicios Ambientales (PSA) son vistos como una de las estrategias de mayor potencial para capturar ingresos que garanticen la gestión de la diversidad biológica presente en estas áreas, así como la provisión de sus servicios ecosistémicos (CBD, 2005; Gutman y Davidson, 2007; MINAM, 2010)3.

De manera complementaria a las ANP que forman parte del sistema nacional a cargo del Servicio Nacional de Áreas Naturales Protegidas por el Estado (Sernanp), el sistema de Áreas de Conservación Regional (ACR) del

3 En el presente documento, el término Pagos por Servicios Ambientales (PSA) es utilizado indistintamente con otros términos como Pagos por Servicios Ecosistémicos, Compensación de Servicios Ambientales y Compensación de Servicios Ecosistémicos. departamento de Loreto es gestionado por el Programa de Conservación, Gestión y Uso Sostenible de la Diversidad Biológica de Loreto (PROCREL) adjunto al respectivo Gobierno Regional. El ACR Alto Nanay-Pintuyacu-Chambira con una extensión de 954,635.48 ha forma parte de este sistema siendo establecida desde el 2011, para conservar los recursos naturales y los ecosistemas frágiles de bosques sobre arena blanca, bosques inundables por aguas negras y bosques de altura, y de esta manera garantizar la provisión de servicios ambientales y el aprovechamiento sostenible de los recursos de flora y fauna silvestre que realizan las poblaciones locales bajo prácticas sostenibles, y promoviendo el desarrollo local y regional. Asimismo, cabe resaltar que se constituye en un espacio de importancia estratégica para los pobladores de la ciudad de lquitos, capital del departamento; pues protege la cabecera de la cuenca que provee de agua potable al 90\% de su población (IIAP, 2009).

En la parte media de la cuenca se encuentra establecida la Reserva Nacional Allpahuayo Mishana (RNAM), perteneciente al sistema nacional y que cuenta con una extensión de poco más de 57 mil ha, de las cuales la mitad están ubicadas en predios privados. La RNAM fue establecida en el 2004 para contribuir a la conservación de ecosistemas como los varillales y chamizales sobre arena blanca, y los bosques inundables por aguas negras, todos ellos endémicos en el área. Asimismo, la iniciativa también busca contribuir a la conservación del recurso hidrológico mediante la promoción del manejo comunal de los recursos presentes.

4 D.S. 005-2011-MINAM 
A pesar de los esfuerzos de conservación antes mencionados, la cuenca durante las últimas décadas ha experimentado procesos de ocupación desordenados, lo que ha derivado en una mayor presión por los recursos naturales presentes (IIAP, 2002; 2009). Este factor en última instancia se ha traducido en mayores tasas de deforestación producto del cambio de uso de suelo para actividades agrícolas, y degradación ambiental como consecuencia de la extracción de madera y otros productos forestales no maderables (PFNM) como el irapay (Lepidocaryum gracile Martius), palmera utilizada en la construcción de galpones para la industria avícola y de viviendas en áreas periurbanas de la ciudad de lquitos ${ }^{5}$.

El objetivo del presente estudio es contribuir al diseño de un mecanismo tipo PSA con énfasis en los servicios de regulación hidrológica provistos por los ecosistemas de la cuenca del Nanay, incluyendo aquellos ubicados en el ACR Alto Nanay-Pintuyacu-Chambira. Las preguntas que se pretende responder en esta investigación son: (1) ¿cuál es la disposición a pagar (DAP) de los usuarios del agua potable de la ciudad de lquitos para conservar los ecosistemas que regulan el flujo hidrológico que ofrece la cuenca del Nanay?; (2) ¿qué factores influencian dicha DAP?, y finalmente (3) ¿qué aspectos deberán de tomarse en cuenta para el establecimiento de un mecanismo tipo PSA?

El resto del documento está estructurado de la siguiente manera: en la siguiente sección

5 De acuerdo a datos no publicados del Programa REDD+ del Ministerio de Ambiente, para el periodo comprendido entre los años 2001 al 2011 en el ámbito de la cuenca se deforestaron en promedio casi $600 \mathrm{Ha}$ anuales. Considerando únicamente el periodo 2008-2011, la tasa promedio de crecimiento de la deforestación ha sido de $50.78 \%$.en promedio. se realizará una revisión de literatura sobre los temas afines al presente artículo, en la sección 3 se detallará la metodología utilizada, para luego mostrarse los resultados en la sección 4. En la sección 5 se discutirán los resultados obtenidos comparándolos con los alcanzados por estudios similares; finalmente las conclusiones son presentadas en la sección 6.

\section{REVISIÓN DE LITERATURA}

La literatura sobre la factibilidad de los PSA como mecanismos de política para la gestión de la diversidad biológica y los servicios ecosistémicos ha sido extensa en los últimos años, particularmente en América Latina y el Caribe (Balvarena et al., 2012). Sin embargo, en el Perú este tipo de trabajos no son numerosos; destacándose por ejemplo el trabajo de Loyola (2007) que explora los aspectos legales, institucionales y económicos para evaluar factibilidad de implementar un esquema de compensación por los servicios ambientales hidrológicos que brinda la cuenca del Chili a las comunidades asentadas a lo largo de ésta.

Dicho estudio incluye la aplicación del método de valoración contingente a los usuarios de los servicios ecosistémicos hidrológicos del río Chili en la ciudad de Arequipa, encontrándose que la disposición a pagar (DAP) por los usuarios de agua potable ascendió a S/. 4.57 (US\$ 1.52) mensuales para el año de estudio 6 .

6 Estimados a un tipo de cambio de S/.3, 00 por cada dólar americano. 
Soncco (2007) valora el servicio ecosistémico de protección del recurso hídrico proporcionado por la cuenca del río Jequetepeque a los beneficiarios establecidos en las cuencas medias y bajas dedicados en su mayoría a la actividad agrícola. La DAP -aplicando valoración contingente con formato referéndum-es estimó en S/. 11.83 (US\$ 3.64); mientras que usando métodos no paramétricos tales como de Turnbull y Kriström fue calculada en $\mathrm{S} /$. 10.41 (US\$ 3.20) y S/. 11.78 (US\$ 3.62), respectivamente.

Respecto a esquemas PSA hidrológicos que ya vienen operando en el ámbito de la Amazonía peruana, el caso más emblemático es la iniciativa para la conservación y regeneración de los ecosistemas llevada a cabo en las subcuencas Mishquiyacu, Ramiyacu y Almendra en el departamento de San Martín que funciona con un aporte ascendente a S/.1.00 (US\$ 0.33) por parte de los usuarios de agua potable en la ciudad de Moyobamba para garantizar la provisión y calidad del agua de consumo. Dicho monto, colectado por la EPS Moyobamba a través de los recibos mensuales del servicio público de agua potable, es destinado para intervenciones específicas que tienen por finalidad minimizar los niveles significativos de cambio de uso de los ecosistemas presentes, y así mantener la diversidad biológica y los servicios ecosistémicos derivados de esta, especialmente aquellos relacionados con la regulación hidrológica (Renner, 2010) $)^{7}$.

Respecto a la experiencia de Moyobamba,

$7 \quad$ Cabe resaltar que el cobro por parte de la EPS mencionada contó con aprobación previa de la Superintendencia Nacional de Servicios de Saneamiento (SUNASS).
Quintero et al. (2009) indican que los resultados de la Herramienta de Evaluación de Suelo y Agua (SWAT, por sus siglas en inglés) aplicado en la cuenca sugieren que los fondos colectados por el PSA podrían servir para incentivar la adopción de sistemas de café bajo sombra con el fin de reducir la sedimentación y a su vez, generar impactos positivos en los medios de vida de los productores establecidos en la parte superior de la cuenca. Es importante mencionar que para implementar la experiencia, el contexto fue favorable dada la alta proporción de personas que estaban dispuestas a contribuir al fondo (82.10\%), y el involucramiento de diversos actores sociales entre autoridades locales y nacionales, así como representantes de la sociedad civil (Aspajo, 2006).

La amplia difusión del PSA en Moyobamba en los últimos años ha venido generando que experiencia similares busquen ser replicadas en otros ámbitos geográficos del Perú, lo que ha llevado a que actualmente se contabilicen más de quince iniciativas tipo PSA en torno a servicios hidrológicos aunque la mayoría aún se encuentra en etapas aún incipientes de consolidación. Por ejemplo, la iniciativa Aquafondo conformada por una coalición de organizaciones públicas y privadas (entre ellas el Ministerio del Ambiente, la cervecería Backus, The Nature Conservancy, entre otras) moviliza fondos para la ejecución de intervenciones de conservación y/o restauración de los ecosistemas en las cuencas de los ríos Chillón, Rímac y Lurín que proveen de agua a Lima y alrededores.

Otras iniciativas pueden ser mencionadas 
como la que se viene desarrollando en la cuenca del río Cañete -con participación también del Ministerio del Ambiente, Care International y el acompañamiento técnico del Centro Internacional de Agricultura Tropical (CIAT)- y la iniciativa en Jaén, ubicado en el departamento de Cajamarca y ejecutado con el acompañamiento de la Cooperación Alemana - GIZ, Cáritas y RARE. Estas experiencias entre otras más, han contribuido a la aprobación de la Ley 30215, "Ley de Mecanismos de Retribución por Servicios Ecosistémicos" en junio del 2014, la cual regula la implementación y funcionamiento de estas iniciativas para garantizar el logro de objetivos ambientales y sociales en el ámbito de su intervención. La norma actualmente se encuentra en etapa de reglamentación para entrar en vigencia.

La viabilidad de este tipo de mecanismos en la Amazonía baja puede verse desfavorecida por la percepción de abundancia del recurso hídrico, factor que explica el poco interés para la implementación de estrategias de conservación de bosques con fines de regulación hidrológica en esta región (Southgate y Wunder, 2007). A lo anterior, se le suma las dificultades para evidenciar empíricamente la relación entre el manejo y conservación de los ecosistemas amazónicos, así también, la provisión y calidad del recurso hidrológico presente.

Sin embargo, Gentry y López-Parodi (1980) muestran que la relación entre deforestación y el desequilibrio de los ciclos hidrológicos son evidenciados por el incremento de precipitaciones en ocho diferentes estaciones meteorológicas en todo el ámbito de la Amazonia peruana. Dichos resultados son congruentes con estudios más recientes como el de Davidson et al. (2012), que reporta el impacto de la expansión agrícola y de la variabilidad climática en los cambios de los ciclos de carbono, nitrógeno y agua en la Amazonía brasilera.

Por su parte, Asquish y Wunder (2008) argumentan que la implementación de un mecanismo tipo PSA se puede establecer incluso bajo condiciones de incertidumbre aceptable, contrario al argumento de la necesidad de contar con evidencia científica completa acerca de los efectos benéficos de determinadas acciones de manejo como una condición inicial indispensable para este tipo de iniciativas. Más importante aún es la presencia de otras condiciones como la existencia de voluntad de pago por parte de los usuarios, y potenciales riesgos en la provisión de los servicios ecosistémicos (Asquish y Wunder 2008). En este sentido, los mecanismos tipo PSA basados en servicios hidrológicos una vez lanzados pueden ser "perfeccionados" incorporando nuevo conocimiento científico, logrando así mayor consenso entre los actores involucrados.

Respecto a la factibilidad de implementación de iniciativas de PSA enfocados en otros servicios ecosistémicos, Armas et al. (2009) exploran el potencial de un esquema de ámbito nacional para la mitigación de emisiones de carbono a partir de la estimación de los costos de oportunidad de las principales actividades económicas causantes de estas emisiones. 
En este caso se encontró que el costo de oportunidad promedio por tonelada de carbono estimado asciende a S/. 13.5 (US\$ 4.5) por tCO2, llegándose a la conclusión que un mecanismo de esta naturaleza tendría un gran potencial de mitigación debido a que la mayoría de las emisiones a nivel nacional son causadas por el cambio de uso del suelo para la práctica agropecuaria de pequeña escala. Smith et al. (1998) llega a las mismas conclusiones aplicando valoración contingente para estimar la disposición a aceptar (DAA) de productores agrícolas en el departamento amazónico de Ucayali por adoptar prácticas agroforestales.

Cranford y Mourato (2011) resaltan la importancia de establecer esquemas de PSA en dos etapas. En la primera, se favorece la creación de un marco de "conservación comunitaria" con vistas a crear un ambiente social adecuado. En la segunda etapa consiste en la implementación en sí del mecanismo de conservación basado en mercados.

Como estudio de caso, se analiza una experiencia de conservación comunitaria de los bosques de Polylepsis en la Coordillera de Vilcanota en el departamento de Cusco. El escenario que se planteó en la valoración consistió en el incremento de la cobertura forestal en las fincas de los productores y sus servicios ecosistémicos (incluyendo regulación y provisión hídrica). El estudio incluye la aplicación de una versión estilizada de métodos basados en preferencias declaradas para estimar el valor social de las acciones de conservación.

\section{METODOLOGÍA}

\subsection{El área de estudio}

La cuenca del Nanay se ubica en su totalidad dentro del departamento de Loreto y cuenta con una extensión de 1721343 ha. En la parte superior de la misma fue establecido el ACR Alto Nanay Pintuyacu Chambira; entre otras razones con el objetivo de proteger las fuentes de agua de este sistema hidrológico que provee de agua a la población urbana de la ciudad de lquitos. Asimismo, en la cuenca media se encuentra establecida la Reserva Nacional Allpahuayo Mishana (Figura 1). La población rural de la cuenca se estima en 30,155 habitantes agrupados en 173 centros poblados entre comunidades (indígenas y campesinas) y caseríos (IIAP, 2009).

La densidad poblacional presente en la cuenca no es uniforme, siendo significativamente mayor en la parte baja de la cuenca dada la cercanía y acceso a la ciudad de lquitos. De acuerdo al IIAP (2009), las actividades económicas predominantes en las partes media y alta se caracterizan por ser eminentemente extractivistas. En ese sentido, el 70,1\% de la población se dedica principalmente a la agricultura, ganadería, caza y silvicultura, y otro $5,7 \%$ a la pesca artesanal; mientras que el restante a actividades como el comercio, servicio doméstico y/o el ejercicio de actividades profesionales (profesores, policías, entre otros).

La agricultura practicada en la cuenca se caracteriza por ser migratoria y de bajos rendimientos, destinándose la mayor parte al autoconsumo y el excedente a los mercados 


\section{Figura 1. Deforestación de la cuenca del Nanay al año 2011}

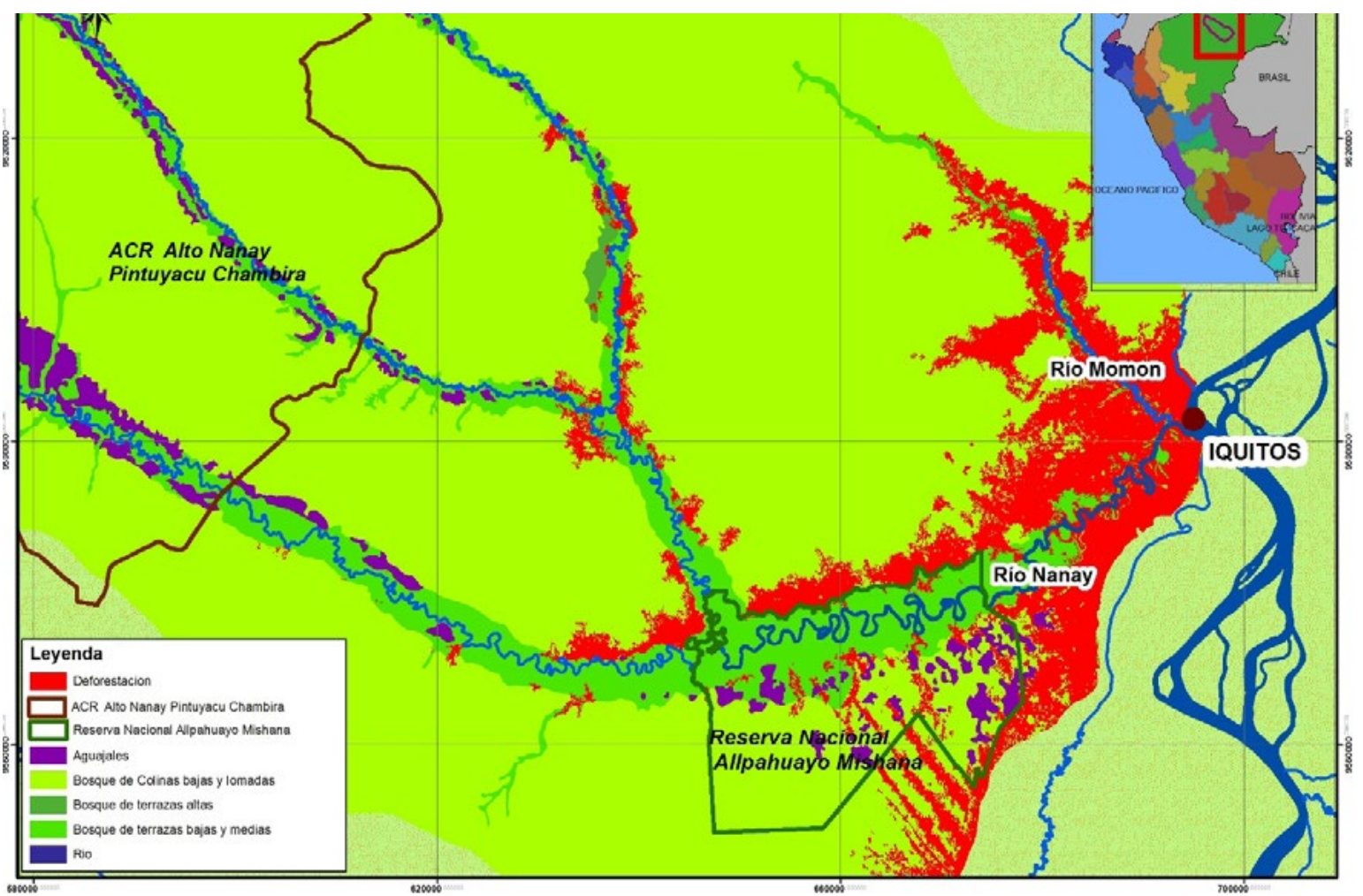

de abastos en la ciudad de lquitos principalmente. Los cultivos anuales más importantes son el plátano, la yuca, el maíz amarillo duro, la caña de azúcar; además de algunos permanentes como el pijuayo, limones, palmito, y frutales como el caimito, la uvilla, la guaba, etc. (IIAP, 2009). En los últimos años se ha incrementado el interés de parte de algunos grupos empresariales de poder acceder a predios ubicados en la cuenca a fin de instalar monocultivos de palma aceitera (Álvarez-Alonso, 2012).

\section{La ciudad de Iquitos}

Iquitos, ubicada al noroeste de Perú, es la capital del departamento de Loreto y la ciudad más poblada de la Amazonía peruana. En el 2011 contaba con 452757 habitantes en un área urbana de $368.9 \mathrm{~km}^{2}$ comprendida por cuatro distritos: Belén, Iquitos, San Juan Bautista y Punchana. Al estar rodeada de ríos, la ciudad sólo cuenta con conexión aérea y fluvial al resto del país, la existencia de carreteras se limita a una que llega hasta Nauta, una pequeña ciudad de aproximadamente $30 \mathrm{mil}$ habitantes ubicada a unos $105 \mathrm{~km}$. El clima de lquitos es tropical, con un rango de temperatura anual que va desde los $15^{\circ} \mathrm{C}$ a $31^{\circ} \mathrm{C}$ (promedio: $23^{\circ} \mathrm{C}$ ) y una humedad relativa promedio del 84\% (INEI, 2008).

\subsection{Muestra}

Para el presente estudio se asume que la población beneficiaria de los servicios hidrológicos de la cuenca son los usuarios del servicio de agua potable en la ciudad de Iquitos; calculándose el tamaño de la muestra mediante la siguiente fórmula (Toma y Rubio 2008): 


$$
n=\frac{k^{2} N p q}{e^{2}(N-1)+k^{2} p q}
$$

Donde: $k$ es el valor tabulado de la distribución normal a un determinado nivel de confianza (para el presente estudio se trabajó con el 95\%); $\mathrm{N}$ es el tamaño de la población bajo estudio (a la fecha del estudio existían 57650 líneas usuarias de agua potable en la ciudad de lquitos.); e el error muestral deseado (se asumió +/- 5\%); y finalmente $\mathrm{p}$ y q la proporción de individuos en la población que poseen o no la característica del estudio, respectivamente (a priori se asume $\mathrm{p}=\mathrm{q}=0.5$ ). Considerando estos parámetros el tamaño mínimo de muestra se estimó en 382 viviendas, sin embargo dados los recursos disponibles para el trabajo inicialmente se convino aplicar un total de 450 encuestas, las cuales fueron distribuidas en cada uno de los distritos que conforman la ciudad de lquitos (Punchana, Belén, San Juan Bautista e lquitos) de acuerdo a la proporcionalidad del número de viviendas usuarias del servicio de agua potable en el respectivo distrito.

Para la selección de las viviendas se aplicó un muestreo aleatorio en dos etapas, el cual será descrito a continuación: en la primera, un plano general de la ciudad de lquitos fue dividido en 60 bloques numerados considerando las áreas que cuentan con el servicio público de agua potable; luego a fin de evitar sesgos y obtener datos provenientes de todas las zonas de la ciudad se seleccionaron hasta 28 bloques de manera aleatoria manteniendo la proporcionalidad en el numero seleccionado de bloques que correspondieron a cada dis- trito. De esta manera, fueron seleccionaron 10 bloques en el distrito de lquitos, 5 bloques en Punchana, 5 en Belén y otros 8 en San Juan Bautista.

En la segunda etapa, seleccionados el número de bloques se distribuyó en partes iguales la cantidad de cuestionarios que debían ser recogidas por cada distrito para estimar a su vez la cantidad de cuestionarios a ser recogidos en cada bloque. En este sentido, en cada bloque seleccionado se planificó recoger entre 16 a 17 encuestas. Para seleccionar cuáles viviendas encuestar en cada bloque, se seleccionó una manzana en el centro del bloque en la cual el encuestador seleccionó una vivienda para posteriormente aplicarse un procedimiento sistemático hasta completar el número de viviendas requerido en cada bloque.

Las encuestas estuvieron dirigidas a los jefes(as) de hogar y/o personas con capacidad de decisión en los gastos familiares y se aplicó de manera personal en visitas a las viviendas de los hogares de la muestra. En el caso de negocios y/o viviendas compartidas, se priorizó la aplicación del cuestionario al responsable del pago del servicio público de agua potable. Es importante mencionar que 20 encuestas fueron descartadas debido a inconsistencias en las respuestas o a no haberse completado la totalidad del cuestionario. Finalmente, se pudieron recoger 158 cuestionarios válidos en lquitos, 72 en Belén, 80 en Punchana y 120 en San Juan Bautista durante el periodo comprendido entre setiembre y octubre del 2011. 
El cuestionario aplicado comprendió tres partes: la primera, cuyo objetivo es la indagación acerca de las percepciones sobre distintos aspectos que aborda la presente investigación, por ejemplo los bosques y sus servicios ecosistémicos (principalmente aquellos relacionados con el recurso hídrico), la importancia de estos en la vida cotidiana y los roles para su conservación. En la segunda parte, se formuló la pregunta de disposición a pago (DAP) bajo el formato referéndum sobre la conservación de los bosques en el área de estudio siguiendo los lineamientos plateados en Arrow et al. (1993), y contándose con ayudas gráficas para explicar los impactos de la deforestación en el ciclo hidrológico.

Finalmente, se recopiló información relacionada con sus aspectos socioeconómicos tales como edad, sexo, origen, número de hijos, grado de instrucción educativa, ingresos familiares, entre otros. Previo a la fase formal de aplicación de encuestas se llevó a cabo una etapa piloto, que implicó la aplicación de 50 cuestionarios, la cual tuvo como objetivos: (i) determinar el vector de precios a proponer; (ii) Mejorar la formulación de algunas preguntas; y (iii) Adiestrar a los encuestadores en la aplicación del tipo de muestreo a utilizado.

\subsection{Estimación de la DAP}

Para la estimación paramétrica, Yi es la DAP, la cual se estimó utilizando especificaciones logit y probit con coeficientes de máxima verosimilitud de acuerdo a la forma:

$$
\operatorname{Prob}\left(\mathrm{Y}_{\mathrm{i}}=1\right)=\alpha_{\mathrm{i}}+\sum_{\mathrm{i}=1}^{\mathrm{k}} \beta_{\mathrm{i}} \mathrm{X}_{\mathrm{i}}+\varepsilon_{\mathrm{i}}
$$

Donde: $\alpha$ es una constante, $\beta$ el vector de coeficientes del modelo, $X$ es el vector de variables regresoras incluyendo un vector de precios estimados a partir de la fase piloto; $y \varepsilon$ el término estocástico. Los valores que conformaron el vector de precios fueron distribuidos uniformemente en los 430 cuestionarios (86 cuestionarios para cada monto propuesto) y aplicados aleatoriamente a la muestra. A fin de evaluar la robustez de las estimaciones, se estimaron tres modelos diferenciados únicamente por la inclusión o no de algunas de las variables presentadas en la tabla 1 bajo ambas especificaciones (logit y probit). Se seleccionó el modelo más adecuado considerando los siguientes criterios: (i) mayor valor log likelihood, (ii) coeficientes significativos al $5 \%$ o $10 \%$ y que tengan el signo esperado; (iii) parámetros Akaike (AIC) y Schwarz (BIC) más bajos en comparación al resto de modelos.

Es importante mencionar que si bien es cierto que la mayoría de estudios de aplicación del método de valoración contingente suelen restringir la DAP hacia valores positivos; en la presente investigación las respuestas de protesto fueron validadas como ceros en la variable dependiente, a fin de poder obtener medidas de la DAP más conservadoras (Halstead et al. 1992).

También se estimó la DAP con los métodos no paramétricos propuestos en Kristöm (1990) y Turnbull (1974); los cuales son útiles cuando se tienen dudas acerca de la distribución de los datos. La media y mediana del DAP bajo estos modelos son determinadas en función de las probabilidades acumuladas de las respuestas (positivas y negativas) de los entre- 


\section{Tabla 1. Variables consideradas en los modelos}

\begin{tabular}{|c|c|c|c|}
\hline Variable & Descripción & Unidad de media & Hipótesis \\
\hline $\begin{array}{l}\text { DAP } \\
\text { (dependiente) }\end{array}$ & $\begin{array}{l}\text { Describe la respuesta del encuestado ante la pre- } \\
\text { gunta si estaría dispuesto a pagar el monto propues- } \\
\text { to para la conservación de los bosques que protegen } \\
\text { el agua consumida en la ciudad de lquitos. }\end{array}$ & $\begin{array}{c}0 \text { =no estaría dispuesto a } \\
\text { pagar } \\
1 \text { =si estaría dispuesto a } \\
\text { pagar }\end{array}$ & - \\
\hline MONTO_PROP & Monto propuesto (en S/.). & $1.00 ; 2.00 ; 5.00 ; 8.00 ; 12.00$ & $\beta<0$ \\
\hline SEXO & Define el género del encuestado. & $\begin{array}{l}0=\text { masculino } \\
1=\text { femenino }\end{array}$ & $\beta>0$ \\
\hline EDAD & Define los años cumplidos del encuestado. & Años & $\beta>0$ \\
\hline EST_ANOS & Años completados de estudios. & Años & $\beta>0$ \\
\hline LABORA_ACT & $\begin{array}{l}\text { Define si el empleado actualmente se encuentra } \\
\qquad \text { empleado. }\end{array}$ & $\begin{aligned} 0 & =\text { No } \\
1 & =\mathrm{Si}\end{aligned}$ & $\beta>0$ \\
\hline HIJOS_CANT & $\begin{array}{l}\text { Define la cantidad de hijos dependientes económica- } \\
\text { mente del encuestado. }\end{array}$ & Continua & $\beta<0$ \\
\hline ING_FAM & $\begin{array}{l}\text { Nivel de ingresos mensuales familiares del en- } \\
\text { cuestado (en S/.). }\end{array}$ & $\begin{array}{c}1=\text { menos de } 600 \\
2=\text { entre } 600 \text { y } 1000 \\
3=\text { entre } 1000 \text { y } 2500 \\
4=\text { entre } 2500 \text { y } 5000 \\
5=\text { entre } 5000 \text { y } 10000 \\
6=\text { =más de } 10000\end{array}$ & $\beta>0$ \\
\hline PERSON_CASA & $\begin{array}{l}\text { Número de personas que viven en la casa del } \\
\text { encuestado. }\end{array}$ & Personas & $\beta>0$ \\
\hline
\end{tabular}

Fuente: Elaboración propia

vistados frente a los precios propuestos.

Finalmente, las DAP calculadas tanto por estimaciones paramétricas como no paramétricas fueron agregadas linealmente para determinar el monto global del fondo que sería destinado a la conservación de los ecosistemas de la cuenca (Riera, 1994).

\section{RESULTADOS}

\subsection{Características socioeconómicas y percepciones}

La muestra estuvo conformada mayoritariamente por mujeres $(67,44 \%)$, las cuales, el $68.60 \%$ tenía entre 30 y 60 años de edad. Respecto al nivel de educación, el $46.74 \%$ de los entrevistados expresa haber seguido hasta algún grado de secundaria, mientras que más de la tercera parte (35.82\%) afirma contar con estudios superiores universitarios y/o no universitarios. Por otro lado, respecto a ingresos familiares el $92.56 \%$ manifiesta contar con ingresos mensuales menores a S/. 2500.00 (US\$ 833.33).

La encuesta incluyó preguntas respecto a las percepciones sobre los servicios hidrológicos 
en cuestión. En este sentido, el $66.28 \%$ conoce cuál es el río que provee de agua potable a la ciudad, y el $44.65 \%$ encontraba que la influencia de los bosques sobre el recurso hídrico es muy importante. Asimismo, una eventual contribución económica para la conservación de los bosques de la cuenca gozaría de la aceptación del 73.95\% de los encuestados.

Se consultó acerca de la competencia de garantizar la conservación de los bosques de la cuenca del Nanay. Los resultados de la encuesta indican que los entrevistados consideran mayoritariamente que la competencia la debe asumir el Gobierno Regional (el $27.44 \%$ ), el $20.7 \%$ consideran que la empresa municipal de agua potable debería hacerse cargo, y otro porcentaje similar considera que es competencia únicamente de los habitantes de la parte superior de la cuenca. La tabla 2 muestra el resumen de los estadísticos descriptivos de las variables consideradas en el modelo.

\section{Tabla 2. Estadísticos descriptivos de las variables consideradas}

\begin{tabular}{|l|c|c|c|c|c|}
\hline \multicolumn{1}{|c}{ Variable } & Obs. & Media & Sta. Dev. & Mín. & Máx. \\
\hline DAP & 430 & 0.34 & 0.47 & 0 & 1 \\
\hline MONTO_PROP & 430 & 5.60 & 4.03 & 1 & 12 \\
\hline SEXO & 430 & 0.67 & 0.47 & 0 & 1 \\
\hline EDAD & 430 & 45.03 & 14.48 & 19 & 94 \\
\hline EST_ANOS & 430 & 11.27 & 3.69 & 0 & 21 \\
\hline LABORA_ACT & 430 & 0.54 & 0.50 & 0 & 1 \\
\hline HIJOS_CANT & $403\left(^{*}\right)$ & 3.08 & 1.79 & 0 & 11 \\
\hline ING_FAM & 430 & 2.17 & 1.03 & 1 & 6 \\
\hline PERSON_CASA & 430 & 5.22 & 2.03 & 1 & 15
\end{tabular}

(*) No considera a hogares sin hijos. Fuente: Elaboración propia

\subsection{Estimación de la DAP}

Los resultados econométricos de los modelos paramétricos logit y probit son presentados en la tabla 3. El modelo I es descartado debido a que la educación del encuestado no presenta el signo esperado y que las variables relacionadas con el nivel de ingreso mensual familiar, el número de personas que viven en la casa y la edad del encuestado no son significativas al $20 \%$. De la misma forma, el modelo III también fue descartado porque presenta parámetros Akaike y Schwarz más altos en comparación al resto de modelos. Por lo tanto, el modelo II es el que mejor satisface los criterios mencionados en la sección metodológica, estimándose un DAP mensual (promedio) por hogar de S/. 1.77 (US\$ 0.59) y S/. 1.72 (US\$ 0.57) bajo las especificaciones logit y probit, respectivamente.

Usando los métodos no paramétricos de Kriström y Turnbull, se estimaron las medianas de la DAP en S/.1.91 (US\$0.64) y S/.1.69 (US\$0.56) mensuales por hogar, respectivamente (véa- 
se el Apéndice 1 para el detalle de las estimaciones realizadas). Posteriormente, los montos obtenidos fueron agregados linealmente, considerando las 57650 conexiones de agua potable existentes para la fecha de realización del presente estudio (Riera, 1994). Los resultados que pueden ser visualizados en la tabla 4.

\section{Tabla 3. Coeficientes estimados por los modelos logit y probit}

\begin{tabular}{|c|c|c|c|c|c|c|}
\hline \multirow{2}{*}{ Variable } & \multicolumn{2}{|c|}{ Modelo I } & \multicolumn{2}{|c|}{ Modelo II } & \multicolumn{2}{|c|}{ Modelo III } \\
\hline & Logit & Probit & Logit & Probit & Logit & Probit \\
\hline CONSTANTE & $\begin{array}{c}0.59 \\
(0.43)\end{array}$ & $\begin{array}{c}0.38 \\
(0.40)\end{array}$ & $\begin{array}{c}0.07 \\
(0.85)\end{array}$ & $\begin{array}{c}0.05 \\
(0.81)\end{array}$ & $\begin{array}{l}0.36^{* *} \\
(0.04)\end{array}$ & $\begin{array}{l}0.21^{*} \\
(0.05)\end{array}$ \\
\hline MONTO_PROP & $\begin{array}{c}-0.20^{\star \star *} \\
(0.00)\end{array}$ & $\begin{array}{c}-0.12^{* *} \\
(0.00)\end{array}$ & $\begin{array}{c}-0.19^{* \star *} \\
(0.00)\end{array}$ & $\begin{array}{c}-0.12^{\star \star *} \\
(0.00)\end{array}$ & $\begin{array}{c}-0.20^{\star * *} \\
(0.00)\end{array}$ & $\begin{array}{c}-0.12^{\star \star \star} \\
(0.00)\end{array}$ \\
\hline SEXO & $\begin{array}{l}0.55^{\star *} \\
(0.04)\end{array}$ & $\begin{array}{l}0.32^{* *} \\
(0.04)\end{array}$ & $\begin{array}{l}0.56^{\star *} \\
(0.03)\end{array}$ & $\begin{array}{l}0.32^{\star *} \\
(0.03)\end{array}$ & & \\
\hline HIJOS_CANT & $\begin{array}{l}-0.10 \\
(0.16)\end{array}$ & $\begin{array}{l}-0.07 \\
(0.13)\end{array}$ & $\begin{array}{l}-0.10 \\
(0.12)\end{array}$ & $\begin{array}{l}-0.06^{*} \\
(0.10)\end{array}$ & & \\
\hline LABORA_ACT & $\begin{array}{c}0.39 \\
(0.11)\end{array}$ & $\begin{array}{l}0.25^{*} \\
(0.09)\end{array}$ & $\begin{array}{l}0.39^{*} \\
(0.08)\end{array}$ & $\begin{array}{l}0.24^{*} \\
(0.08)\end{array}$ & & \\
\hline PERSON_CASA & $\begin{array}{c}0.02 \\
(0.71)\end{array}$ & $\begin{array}{c}0.01 \\
(0.70)\end{array}$ & & & & \\
\hline ING_FAM & $\begin{array}{c}0.16 \\
(0.25)\end{array}$ & $\begin{array}{c}0.10 \\
(0.21)\end{array}$ & & & & \\
\hline EST_ANOS & $\begin{array}{l}-0.06 \\
(0.11)\end{array}$ & $\begin{array}{l}-0.04^{*} \\
(0.09)\end{array}$ & & & & \\
\hline EDAD & $\begin{array}{l}-0.01 \\
(0.56)\end{array}$ & $\begin{array}{l}-0.03 \\
(0.58)\end{array}$ & & & & \\
\hline Log Likelihood & -230.43 & -230.45 & -232.26 & -232.26 & -250.14 & -250.41 \\
\hline Chi2 (C2) & 51.78 & 57.13 & 48.83 & 48.83 & 40.24 & 43.98 \\
\hline $\mathrm{AIC}$ & 478.86 & 478.91 & 474.52 & 474.52 & 504.28 & 504.83 \\
\hline $\mathrm{BIC}$ & 514.85 & 514.90 & 494.52 & 494.52 & 512.41 & 512.96 \\
\hline Media DAP (S/.) & 1.79 & 1.77 & 1.77 & 1.72 & 1.80 & 1.75 \\
\hline $\begin{array}{l}\text { Intervalo de } \\
\text { Confianza }\end{array}$ & {$[1,59 ; 1,99]$} & {$[1,57 ; 1.98]$} & {$[1.59 ; 1.94]$} & {$[1.55 ; 1.90]$} & - & - \\
\hline
\end{tabular}

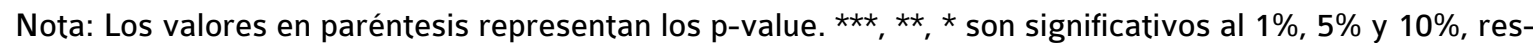
pectivamente. Todas las estimaciones fueron corregidas por problemas de heteroscedasticidad utilizando los errores estándar robustos de Hubert-White. Fuente: Elaboración propia

Otro aspecto consultado fue sobre el vehículo de pago a través del cual se haría este cobro, frente a lo cual la amplia mayoría de encuestados $(75.40 \%)$ respondieron que el pago se haga a través del recibo de agua, mientras que otro porcentaje menor (12.78\%) prefiere que sea a través de arbitrios municipales. Asimismo, se indagó acerca de las razones por las que los encuestados no están de acuerdo con una iniciativa de esta naturaleza, obteniéndose como respuestas más frecuentes aquellas relacionadas a razones económicas (35.04\%), desconfianza en las instituciones y/o corrupción (22.22\%), y que el Estado debería hacerse cargo (20.51\%). 


\section{Tabla 4. Agregación de las DAP obtenidas}

\begin{tabular}{|c|c|c|c|c|c|c|}
\hline \multirow{2}{*}{ Modelo } & \multicolumn{2}{|c|}{ Por hogar mensual } & \multicolumn{2}{|c|}{ Por hogar anual } & \multicolumn{2}{|c|}{ Total agregado anual } \\
\hline & $\mathbf{S} /$. & US\$ & $\mathbf{S} /$. & US\$ & $\mathbf{S} /$. & US\$ \\
\hline \multicolumn{7}{|l|}{ Paramétricos $\left(^{*}\right)$} \\
\hline $\begin{array}{l}\text { Logit } \\
\text { (media) }\end{array}$ & 1.77 & 0.59 & 21.24 & 7.08 & 1224486 & 408162 \\
\hline $\begin{array}{l}\text { Probit } \\
\text { (media) }\end{array}$ & 1.72 & 0.57 & 20.64 & 6.88 & 1189896 & 396632 \\
\hline \multicolumn{7}{|l|}{ No paramétricos } \\
\hline $\begin{array}{l}\text { Kriström } \\
\text { (mediana) }\end{array}$ & 1.91 & 0.64 & 22.92 & 7.64 & 1321338 & 440446 \\
\hline $\begin{array}{l}\text { Turnbull } \\
\text { (mediana) }\end{array}$ & 1.69 & 0.56 & 20.28 & 6.76 & 1169142 & 389714 \\
\hline
\end{tabular}

(*) Las DAP obtenidas mediante métodos paramétricos presentadas corresponden al modelo II estimado en la tabla 3. Fuente: Elaboración propia

\section{DISCUSIÓN}

Las DAP obtenidas en el presente estudio son sustancialmente menores a las reportadas en Quintero et al. (2005), Loyola (2007) y Soncco (2007) en el diseño de iniciativas de PSA locales basados en el recurso hídrico a implementarse en otros contextos geográficos en el Perú. Las diferencias se deberían básicamente a dos razones: la primera el contexto en el cual se realizó la valoración, en este sentido tal como lo plantean Southgate y Wunder (2006), la abundancia del agua en la región amazónica así como la demanda relativamente pequeña por los servicios hidrológicos comparada con la de otras áreas de la costa o sierra limitan el interés por la implementación de este tipo de iniciativas en el área de estudios.

La segunda razón, del tipo metodológico, se debe a la inclusión de DAP estimados con signo negativo como consecuencia de incluir las respuestas de protesta como ceros frente a los precios propuestos durante la aplicación del cuestionario. Esto se realizó basándonos en el tratamiento propuesto por Halstead et al. (1992) que argumentan que tal práctica puede ser aplicable en ejercicios de evaluaciones de políticas más que en la cuantificación del valor del servicio ecosistémico en sí, tal como el que se aborda en el presente estudio. En este sentido, las DAP así obtenidas brindan medidas conservadoras y están íntimamente ligadas también al nivel del aceptación de la política propuesta en sí (Jorgensen y Syme 2000). La aplicación de esta lógica puede ser revisada en Jakobsson y Dragun (1996) y en Hanley et al. (2008) ${ }^{8}$.

En cuanto a las especificaciones paramétricas, el modelo II seleccionado tiene como variables que afectan la DAP al monto propuesto, el sexo del entrevistado, la cantidad de hijos dependientes en el hogar y si la persona se encontraba empleado de manera estable en el tiempo de aplicación del cuestionario (mo-

8 Algunos autores como Carson et al. (1992) y Bateman et al. (2002) recomiendan el truncamiento de valores negativos en la DAP, procedimiento que es aplicado ampliamente en los estudios de valoración contingente. 
delada con la variable binaria LABORA_ACT). Dichas variables cuentan con el signo esperado (véase la columna hipótesis de la tabla 1) y tienen base en literatura previa que sustenta su inclusión en el modelo final seleccionado.

Por ejemplo, el efecto del género del entrevistado en la DAP se presenta como uno de los más significativos en el presente estudio. Argumentos tales como una mayor actitud de protección de la naturaleza presente en las mujeres debido a su adiestramiento desde la infancia como futuras responsables del bienestar del hogar; y la actitud socialmente asumida por los varones para ser el soporte económico de la familia llevándolos a preocuparse más por contar con ingresos económicos para sustentar los gastos del hogar aun a expensas del ambiente, son los que han llevado tradicionalmente a plantear hipótesis que relacionan el género del entrevistado con ciertas actitudes, creencias y acciones relacionadas con la conservación ambiental (Stern et al,. 1993). Sin embargo, tales hipótesis en la mayoría de casos no son concluyentes, y por el contrario algunos estudios que los plantean presentan resultados contradictorios (Teal y Loomis, 2000). En este sentido, los resultados del presente estudio aportan evidencia empírica a favor de una mayor predisposición presente en las mujeres para participar en iniciativas de conservación de la naturaleza en el ámbito de estudio.

Uno de los resultados más interesantes evidenciados en el presente estudio ha sido la baja significancia de los ingresos familiares en el modelo I. Si bien el efecto de los ingresos sobre la DAP ha sido ampliamente discutido en la literatura especializada (Hanemman, 1991; Horowitz y McCollel, 2003), el efecto en esta medida de bienestar por la pérdida de los ingresos esperados como consecuencia de la implementación de las iniciativas propuestas en el escenario contingente ha sido menos estudiado. Por ejemplo, cuando una iniciativa consistente en el establecimiento de un área protegida podría generar pérdidas futuras de ingresos provenientes por aprovechamiento forestal en la zona. Sobre este último aspecto, Hackl y Prucker (2006) mencionan la posibilidad de estimar DAP sesgadas si es que tales situaciones no son advertidas en el marco del estudio y proponen la inclusión de diversas preguntas de control que minimicen tal distorsión, y que sirvan además como variables alternativas en los posteriores análisis econométricos. Considerando tal aspecto, en nuestro modelo paramétrico se ha optado por incluir la variable binaria LABORA_ACT que captura el efecto sobre la DAP de contar con ingresos estables, que presenta significancia al $10 \%$.

La cantidad de hijos dependientes económicamente en el hogar es otra de las variables consideradas en el modelo debido a que presenta el signo esperado negativo y tiene nivel de significancia cercano al 10\%. La manera de cómo esta variable influye sobre la DAP es instintiva: el mayor número de hijos dependientes conlleva mayores gastos por lo cual el consumo de otros bienes o servicios se ve limitado. Loyola (2007) reporta el efecto significativo de la misma variable en la valoración realizada en la cuenca del río Chili, en la costa sur peruana. 
Las estimaciones no paramétricas realizadas en el presente estudio demuestran la influencia del vector de precios seleccionados en las estimaciones de la DAP (Cerda y Vásquez, 2005). Particularmente en el método de Kriström la estimación de la medida del bienestar depende en gran medida del valor que equivale a la probabilidad de $0 \%$ de respuestas afirmativas, el cual se reconoce es un proceso arbitrario (Rodríguez, 2000). En este sentido, en el presente estudio al precio mayor establecido (S/.12.00 o US\$ 4.00) aún le correspondía una frecuencia acumulada importante de aceptación (13.95\%), lo cual se tradujo un alto aporte de este último tramo de la demanda (equivalente a S/. 1.88 o US\$ 0.63) en el promedio de la DAP. Debido a ello, se optó por utilizar las medianas debido que su uso es recomendado en caso la distribución presente problemas de asimetría; como cuando la amplia mayoría de la población estaría dispuesta a pagar montos bastante menores, y una proporción más pequeña de la misma estaría dispuesta a pagar montos bastante mayores (Harrison y Kriström, 1995; Gutiérrez-Barrera, 2002). Las medianas estimadas mediante las técnicas no paramétricos de Kriström (S/. 1.91 o US\$ 0.64) y Turnbull (S/. 1.69 o US\$ 0.56) son coherentes con las DAP estimadas por el modelo paramétrico.

Los resultados arrojan la posibilidad que implementación de un mecanismo tipo PSA en Iquitos ubicado en la Amazonía baja peruana, el mismo que pudiera complementar la iniciativa gubernamental de conservación comunal que ya viene llevándose a cabo en la cuenca con el fin de mejorar su efectividad (Minang y van Noordwijk, 2013). En este sen- tido, los próximos pasos para el diseño de la iniciativa podrían enfocarse en:

1. Analizar la oferta de los servicios ecosistémicos de la cuenca, considerando la posibilidad de que muchos de estos podrían estar íntimamente vinculados (Asquith et al. 2008; Engel et al. 2008). Tal análisis debe considerar la inclusión de los actores coadyuvantes de estos servicios presentes en la cuenca (principalmente comunidades campesinas e indígenas), estableciendo objetivos de desarrollo social (Greiner y Stanley 2013) y de acciones que contribuyan a los esfuerzos de adaptación al cambio climático (van de Sand 2012) dada la vulnerabilidad del área de intervención en ambos aspectos.

2. Explorar aspectos institucionales $y$ normativos para la implementación de la iniciativa. En este sentido, dos leyes recientemente aprobadas: la Ley 30045 "Ley de Modernización de los Servicios de Saneamiento" y la Ley 30215 "Ley de Mecanismos de Retribución por Servicios Ecosistémicos", resultan claves para la construcción de una adecuada plataforma institucional que brinde soporte jurídico a la presente iniciativa.

3. La implementación en sí, la cual se sugiere primero deberá iniciar con un proceso de concientización (Cranford y Mourato, 2011). La aplicación de un plan de comunicaciones acerca de los beneficios de la iniciativa podría contribuir en este proceso. 


\section{CONCLUSIONES}

La percepción de abundancia del recurso hídrico en el área de estudio influye sobre las DAP estimadas que son relativamente bajas comparándolas con estudios de valoración realizados en otros contextos nacionales. Asimismo, las medidas de bienestar estimadas por métodos paramétricos y no paramétricos en el presente estudio son coherentes; constituyéndose estos últimos en un buen soporte metodológico para confirmar la robustez de las estimaciones econométricas.

De acuerdo al modelo econométrico seleccionado, las variables que determinan la DAP por la conservación de los ecosistemas de la cuenca del Nanay por parte de los usuarios del servicio público de agua potable en Iquitos son el precio propuesto, el sexo, número de hijos dependientes económicamente y si alguno de los jefes del hogar se encontraba empleado al momento de aplicación del cuestionario.

Las DAP calculadas agregadas arrojan la posibilidad de que eventualmente se pueda conformar un fondo a partir de las contribuciones de los usuarios de agua potable de la ciudad de lquitos para complementar y mejorar la efectividad de la iniciativa de conservación comunal ya establecida en la cuenca del Nanay. 


\section{REFERENCIAS BIBLIOGRÁFICAS}

Álvarez-Alonso, J. 2012. ¿Monocultivos en el Nanay? No, gracias. Diario La Región del 21 de abril del 2012 (en línea). Consultado el 02 de marzo del 2013. Disponible en: http://diariolaregion.com/web/2012/04/21/monocultivos-en-el-nanay-no-gracias/.

Armas, A; Börner, J; Rügnitz Tito, M; Díaz, L; Tapia, S; Wunder, S; Reymond, L; Nascimento, N. 2009. Pagos por Servicios Ambientales para la conservación de bosques en la Amazonía Peruana. SERNANP. 78 p.

Arrow, K; Solow, R; Portney, P; Leamer, EE; Radner, R; Schuman, H. 1993. Report of the NOAA Panel on Contingent Valuation. Registro Federal 58(10): 4602-4614.

Aspajo, F. 2006. Mecanismo de Pago por Servicios Ambientales en la Ciudad de Moyobamba. Sistematizando las experiencias del Proyecto Cuencas Andinas. Centro Internacional de la Papa (CIP). 29 p.

Asquith, N; Vargas, MT; Wunder, S. 2008. Selling two environmental services: In-kind payments for bird habitat and watershed protection in Los Negros, Bolivia. Ecological Economics 65(2008): 675-684.

Asquith, N; Wunder, S (Eds). 2008. Payments for Watershed Services: The Bellagio Conversations. Fundación Natura Bolivia, Santa Cruz de la Sierra. $32 \mathrm{p}$.
Ayer, M.; Brunk, HD.; Ewing, GM.; Reid, WT.; Silverman, E. 1955. An Empirical Distribution Function for Sampling with Incomplete Information. The Annals of Mathematical Statistics, 26(4), 641-647.

Balvanera, P; Uriarte, M; Almeida-Leñero, L; Altesor, A; DeClerck, F; Gardner, T; Hall, J; Lara, A; Laterra, P; Peña-Claros, M; Silva-Matos, D; Vogl, A; Romero-Duque, LP; Arreola, LF; Caro-Borrero, AP; Gallego, F; Jain, M; Little, C; de Oliveira-Xavier, R; Paruelo, JM, Peinado, JE; Poorter, L. 2012. Ecosystem services research in Latin America: The state of the art. Ecosystem Services 2: 56-70.

Bateman, IJ.; Carson RT; Brett D.; Hanenmann M.; Hanley N.; Hett T.; Jones-Lee M.; Loomes G.; Mourato S.; Özdemiroğlu. E.; Pearce D.; Sudgen, R.; Swanson, J. 2002. Economic Valuation with Stated Preferences Techniques: A Manual. Edward Elgar Publications. Cheltenhan, UKNorthampton, MA.

Carson, RT.; Mitchell, R.; Hanemann, M.; Kopp, RJ.; Presser, S.; Ruud, PA. 1992. A contingent valuation study of lost passive use values resulting from the Exxon Valdez oil spill: a report to the Attorney General of the State of Alaska (Vol. 1): State of Alaska Attorney General's Office.

CBD.2005. Options for mobilizing financial resources for the implementation of the programme of work by developing countries and countries with economies in transition. Grupo de Trabajo sobre Áreas Protegidas. Primera Reunión. UNEP/CBD/WG-PA/1/3. 
Cerda, A.; Vásquez, F. (2005). Differences between parametric and non-parametric estimation of welfare measures: An application to the Rio Claro, Talca, Chile. Panorama socioeconómico (31), 22-31.

Cranford, M; Mourato, S. 2011. Community conservation and a two-stage approach to payments for ecosystem services. Ecological Economics 71: 89-98.

Davidson, E; de Araújo, A; Artaxo, P; Balch, JK; Brown, IF; Bustamante, M; Coe, MT; DeFries, RS; Keller, M; Longo, M; Munger, JW; Schroeder, W; Soares-Filho, B; Souza Jr, CM; Wofsy, SC. 2012. The Amazon Basin in Transition. Nature 481: 321-328.

Engel, S; Pagiola, S; Wunder, S. 2008. Designing payments for environmental services in theory and practice: An overview of the issues. Ecological Economics 65(2008): 663674.

Fearnside, P.M. 1997. Environmental services as a strategy for sustainable development in rural Amazonia. Ecological Economics, 20(1), 53-70.

Gentry, AH; Lopez-Parodi, J. 1980. Deforestation and Increased Flooding of the Upper Amazon. Science 210: 1354-1356.

Greiner, R; Stanley, O. 2013. More than money for conservation: Exploring social co-benefits from PES schemes. Land Use Police 31 (2013): 4-10.
Gutiérrez-Barrera, J. 2002. Valoración económica del servicio ambiental hídrico en las sub cuencas Molino Norte y San Francisco, y propuesta para su incorporación en la tarifa hídrica, Matagalpa, Nicaragua. Tesis MSc. CATIE. Turrialba, CR. 117p.

Gutman, P; Davidson, S. 2007. A review of innovative international financial mechanisms for biodiversity conservation with a special focus on the international financing of Developing Countries' Protected Areas. Informe para Programa WWF-MPO. $68 \mathrm{p}$.

Haab, T; McConnell, KE. 2002. Valuing environmental and natural resources: the econometrics of non-market valuation: Edward Elgar Publishing.

Hackl, F.; Pruckner, GJ. 2006. Do expected income changes bias contingent valuation wiIlingness-to-pay figures? Quarterly Journal of International Agriculture, 45(4), 421.

Hanemann, WM. 1991. Willingness to Pay and Willingness to Accept: How Much Can They Differ? The American Economic Review, 81(3), 635-647.

Halstead, JM; Luloff, A; Stevens, TH. 1992. Protest bidders in contingent valuation. Northeastern Journal of Agricultural and Resource Economics, 21(2), 160-169.

Hanley, N.; Colombo, S.; Kriström, B.; Watson, F. 2009. Accounting for Negative, Zero and Positive Willingness to Pay for Landscape Change in a National Park. Journal of Agricul- 
tural Economics, 60(1), 1-16.

Hardner, J. 2008. The Problem of Financing Protected Areas in the Andes-Amazon Region. Economics and Conservation in the Tropics: A Strategic Dialogue. Conference Paper. $8 \mathrm{p}$.

Harrison, GW; Kriström, B. 1995. On the interpretation of responses in contingent valuation surveys En Johansson, PO; Kriström, B; Mäler, KG (Eds). Current issues in Enviromental Economics Manchester University Press, Manchester, GB.

Horowitz, JK.; McConnell, KE. 2003. Willingness to accept, willingness to pay and the income effect. Journal of Economic Behavior \& Organization, 51(4), 537-545.

INEI. 2008. Perú Compendio Estadístico 2008. Sistema Estadístico Nacional.

IIAP. 2002. Propuesta de Zonificación Ecológica Económica de la Cuenca del Río Nanay - Medio Socioeconómico (Vol. IV) Programa de Ordenamiento Territorial-POA (pp. 61). Iquitos, Perú.

IIAP. 2009. Diagnóstico y marco estratégico para la Gestión Integrada de la cuenca del río Nanay, Loreto (Documento en consulta). Foro Peruano para el Agua. 109 p.

Jakobsson, KM; Dragun, AK. 1996. Contingent valuation and endangered species: methodological issues and applications: Edward Elgar Publishing.
Jorgensen, BS; Syme, GJ. 2000. Protest responses and willingness to pay: attitude toward paying for stormwater pollution abatement. Ecological Economics, 33(2), 251-265.

Kriström, B. 1990. A Non-Parametric Approach to the Estimation of Welfare Measures in Discrete Response Valuation Studies. Land Economics 66 (2): 135-139.

Loyola, R. 2007. Valoración del servicio ambiental de provisión de agua con base en la Reserva Nacional Salinas y Aguada Blanca Cuenca del Río Chili. PROFONANPE. 228 p.

MINAM. 2010. Conversatorio Internacional Mecanismos de Financiamiento para la Conservación de los Ecosistemas y la Biodiversidad. $136 \mathrm{p}$.

Minang, P; van Noordwijk, M. 2013. Design challenges for achieving reduced emissions from deforestation and forest degradation through conservation: Leveraging multiple paradigms at the tropical forest margins. Land Use Policy 31(2013): 61-70.

Quintero, M; Estrada RD; Girón E. 2005. Mecanismos de pago por servicios ambientales en la Región de Moyobamba. Estudio Piloto: Microcuencas Miskiyacu y Rumiyacu. Proyecto Cuencas Andinas. 27 p.

Quintero, M; Wunder, S; Estrada, RD. 2009. For services rendered? Modeling hydrology and livelihoods in Andean payments for environmental services schemes. Forest Ecology and Management 258: 1871-1880. 
Renner, I. 2010. Compensation scheme for upstream farmers in municipal protected area, Peru. The Economic of Ecosystems and Biodiversity (TEEB). $2 \mathrm{p}$.

Riera, P. 1994. Manual de Valoración Contingente. Instituto de Estudios Fiscales. 112 p.

Rodriguez, DJ. 2000. Cost-Benefit analysis of environmental quality improvement projects: uncertain benefits of willingness to pay from referendum contingent valuation. Tesis M.A. Virginia Polytechnic Institute and State University.

SERNANP. 2009. Plan Financiero del SINANPE. $93 \mathrm{p}$.

Smith, J., Mourato, S., Veneklaas, E., Labarta, R., Reategui, K., \& Anches, G. (1998). Can global environmental markets help to control tropical deforestation? Evidence from the Peruvian Amazon. Paper presentado en el First World Congress on Environmental and Resource Economics, Venice, Italy. 36 p.

Soncco. C. 2007. Valoración Económica del Servicio Ambiental de Protección del Recurso Hídrico. Estudio de caso de la cuenca del río Jequetepeque Cajamarca - La Libertad, Perú. SEPIA XII Perú: El Problema Agrario en Debate (en línea). Consultado el 01 de marzo del 2013. Disponible en: http://joseordinolaboyer.files.wordpress.com/2011/01/valoracion economica_servicio_ambiental_cajamarca_ la_libertad_c-soncco.pdf.

Southgate, D; Wunder, S. 2009. Paying for Wa- tershed Services in Latin America: A Review of Current Initiatives. Journal of Sustainable Forestry 28: 497-524.

Stern, PC.; Dietz, T.; Kalof, L. 1993. Value Orientations, Gender, and Environmental Concern. Environment and Behavior, 25(5), 322-348.

Teal, GA.; Loomis, JB. (2000). Effects of Gender and Parental Status on the Economic Valuation of Increasing Wetlands, Reducing Wildlife Contamination and Increasing Salmon Populations. Society \& Natural Resources, 13(1), 1-14.

Toma, J.; Rubio, J. 2008. Estadística aplicada. Segunda Parte. CIUP (Centro de Investigaciones de la Universidad del Pacífico). Serie: Apuntes de Estudio 69. Lima, Perú.

Turnbull, B. 1974. The Empirical Distribution Function with Arbitrarily Grouped, Censored and Truncated Data. Journal of the Royal Statistical Society. Series B (Methodological) 38(3): 209-295.

van de Sand, I. 2012. Payments for Ecosystem Services in the Context of Adaptation to Climate Change. Ecology and Society 17(1):11. 


\section{APENDICE 1: ESTIMACIÓN NO PARAMÉTRICA DE LA DAP}

Primero describimos el modelo no paramétrico presentado en Kriström (1990). Se parte de una función de proporciones de respuestas positivas generada a partir de las respuestas discretas recogidas de la aplicación de la pregunta sobre la DAP en formato referéndum:

$$
\dot{\pi}=\left(\dot{\pi}_{1}, \dot{\pi}_{2}, \dot{\pi}_{3} \ldots \dot{\pi}_{m}\right)
$$

Donde convenimos que $\dot{\pi}_{1}$ corresponde al precio propuesto más bajo $A_{1}, \dot{\pi}_{2}$ al siguiente más bajo $A_{2^{\prime}}$ y así sucesivamente. Ayer et al. (1955) demuestran que si $\dot{\pi}$ está conformada por una secuencia de proporciones monótona y no incremental entonces esta secuencia podría proveer un estimador para la probabilidad de aceptación de máxima verosimilitud de distribución libre.

Si la secuencia no es monótona entonces se plantea el siguiente algoritmo: Si $\dot{\pi}_{i}<\dot{\pi}_{i+1}$ para algún $i(i=1,2, \ldots ., \mathrm{m}-1)$, entonces $\overline{\pi_{1}}=\overline{\pi_{l+1}}$ donde la barra denota el estimador de máxima verosimilitud. Las proporciones $\dot{\pi}_{i}$ y $\dot{\pi}_{i+1}$ son reemplazados por: $\left(k_{i}+\right.$ $\left.k_{i+1}\right) /\left(n_{i}+n_{i+1}\right)$. Y así el procedimiento es repetido hasta que la secuencia sea monótona en i. Ayer et al. (1955) demuestran que este estimador tiene condiciones deseables de consistencia. A partir de la regla anterior queda demostrado que pueden haber hasta $m$ estimados de la probabilidad de aceptación, por lo cual se necesita una regla para interpolarse entre estos puntos. Por ejemplo,
Kriström (1990) utiliza la interpolación lineal.

Al igual que el procedimiento anterior, el procedimiento planteado en Turnbull (1974) permite el cálculo de un estimador de máxima verosimilitud para estudios de valoración de preferencias declaradas (Haab y McConell 2002). En este sentido, considere la típica pregunta de valoración contingente ¿Estaría usted dispuesto a pagar una cantidad $b_{j}$ ?. El monto $b_{j}$ donde $j=0,1, \ldots ., \mathrm{M}+1$ y $b_{j}>b_{k}$ para $j>k$, y $b_{0}=$ 0 . Además, sea $p_{j}$ la probabilidad que el monto de DAP de la persona encuestada se encuentre en el intervalo de monto de $b_{j-1}$ a $b_{j}$. Esto se puede escribir de la siguiente manera:

$$
p_{j}=P\left(b_{j-1}<w<b_{j}\right) \text { para } j=1,2, \ldots M+1
$$

Alternativamente, la función de distribución acumulada se define:

$F_{j}=P\left(W<b_{j}\right)$ para $j=1,2, \ldots M+1$, donde $F_{M+1}=1$

La idea es lograr un bj lo suficientemente alto que permita que $F_{M+1}=1$. Entonces:

$$
p_{j}=F_{j}-F_{j-1}
$$

y $F=0$. El estimador Turnbull pude ser calculado tratando a $F_{j}$ cuando $j=1 \ldots M$, o a $p_{j^{\prime}}$ $j=1$...M como parámetros.

A partir del proceso planteado en Rodríguez (2000), a continuación se detalla paso a paso el cálculo de la DAP con datos del presente estudio mediante los métodos no paramétricos mencionados en la presente sección. 


\section{Estimación de la DAP mediante el método no paramétrico de Kriström}

\begin{tabular}{|l|c|c|c|c|c|c|}
\hline $\begin{array}{c}\text { Gru- } \\
\text { po }\end{array}$ & $\begin{array}{c}\text { Monto (S/. por } \\
\text { mes) }\end{array}$ & Rango & $\begin{array}{c}\text { Punto } \\
\text { medio }\end{array}$ & $\begin{array}{r}\text { Proporción } \\
(\mathbf{\%}) \text { respuestas } \\
\text { positivas }\end{array}$ & $\mathbf{P}_{\mathbf{j}}=\mathbf{F}_{\mathbf{j}-1}-\mathbf{F}_{\mathbf{j}}$ & $\begin{array}{c}\text { Estimado de } \\
\text { la DAP }\end{array}$ \\
\hline $\mathrm{Na}$ & 0 & 0 & $\mathrm{Na}$ & 1.00 & na & 0.00 \\
\hline 0 & 1 & $0-1.00$ & 0.5 & 0.6046512 & 0.395349 & 0.1976744 \\
\hline 1 & 2 & $1.00-2.00$ & 1.5 & 0.4534884 & 0.151163 & 0.2267442 \\
\hline 2 & 5 & $2.00-5.00$ & 3.5 & 0.3023256 & 0.151163 & 0.5290698 \\
\hline 3 & 8 & $5.00-8.00$ & 6.5 & 0.2093023 & 0.093023 & 0.60465145 \\
\hline 4 & 12 & $8.00-12.00$ & 10 & 0.1395349 & 0.069767 & 0.697674 \\
\hline 5 & 15 & $12.00-15.00$ & 13.5 & 0.00 & 0.1395349 & 1.88372115 \\
\hline
\end{tabular}

Fuente: Elaboración propia

Para el cálculo de la mediana se interpoló linealmente sobre los precios propuestos (inferior y superior) relacionados con la función de frecuencias acumuladas por encima y por debajo al $50 \%$ de respuestas positivas. La fórmula aplicada es $M e d=b_{u}-k^{*} i$ donde $b_{u}$ es el monto de la clase superior que contiene el $50 \%$ de respuestas positivas (en este caso
$\mathrm{S} / .2 .00), i$ es el rango de clases que contiene el $50 \%$ de respuestas positivas $(1=2.00-$ 1.00) y $k$ el punto donde aproximadamente el $50 \%$ de las observaciones se intercepta [ 1 $0.4535)-0.50) /(0.6047-0.4535)]$. Entonces $\mathrm{Med}=2-1 * 0.085=S / .1 .91$ (US\$ 0.64) por mes por hogar.

\section{Estimación de la DAP mediante el método no paramétrico de Turnbull}

\begin{tabular}{|l|c|c|c|c|c|c|}
\hline Grupo & $\begin{array}{c}\text { Monto (S/. por } \\
\text { mes) }\end{array}$ & Rango & $\begin{array}{c}\text { Límite } \\
\text { inferior }\end{array}$ & $\begin{array}{c}\text { Proporción } \\
\text { respuestas } \\
\text { negativas }\end{array}$ & $\mathbf{P}_{\mathbf{j}}=\mathbf{F}_{\mathbf{j}-1}-\mathbf{F}_{\mathbf{j}}$ & $\begin{array}{c}\text { Estimado } \\
\mathbf{d e} \text { la DAP }\end{array}$ \\
\hline 0 & 1 & $(0-1)$ & 0 & 0.3953488 & na & 0 \\
\hline 1 & 2 & $(1-2)$ & 1 & 0.5465116 & 0.151163 & 0.1511628 \\
\hline 2 & 5 & $(2-5)$ & 2 & 0.6976744 & 0.151163 & 0.3023256 \\
\hline 4 & 8 & $(5-8)$ & 5 & 0.7906977 & 0.093023 & 0.4651165 \\
\hline 5 & 12 & $(8-12)$ & 8 & 0.8604651 & 0.069767 & 0.5581392 \\
\hline & $>12$ & $(12$ a más) & 12 & 1 & 0.139535 & 1.6744188 \\
\hline & & & & & Media de la DAP (S/.) & 3.1511629 \\
\hline
\end{tabular}

Fuente: Elaboración propia

Similar al anterior, para calcular la mediana se aplicó la fórmula: $M e d=b_{u}+k^{*} i$ donde bu es el monto de la clase inferior que contiene el $50 \%$ de respuestas negativas (en este caso $\mathrm{S} / .1 .00), i$ es el rango de clases que contiene el $50 \%$ de respuestas negativas $(1=2.00$
- 1.00) y $k$ el punto donde aproximadamente el $50 \%$ de las observaciones se intercepta [(0.50-0.3953) / (0.5465-0.3953)]. Entonces $\mathrm{Med}=1+1^{*} 0.69=S / .1 .69$ (US\$ $0.56)$ por mes por hogar. 
Finalmente, dado que las medias estimadas por ambos métodos no paramétricos reflejan inconsistencias que se deben al vector de precios seleccionados (Cerda y Vásquez, 2005), optaremos por realizar las agregaciones de la DAP con las medianas obtenidas, pues se considera que las mismas representan de mejor manera las preferencias en el área de estudio; además resulta en una medida más adecuada en situaciones donde la DAP presenta distribución asimétrica (Harrison y Kriström, 1995).

\section{RECONOCIMIENTOS Y AGRADECIMIENTOS}

Los datos obtenidos para el presente artículo se recopilaron en el marco de la consultoría Elaboración de una Propuesta de Compensación por los Servicios Ecosistémicos Hídricos en la Cuenca del Río Nanay convocada por el Consorcio NCI-IIAP con financiamiento de la Fundación Moore durante el año 2011. Agradecemos a Eduardo Rojas Báez por su apoyo en la elaboración del mapa del área de estudio. 